UTPT-96-16

\title{
Stochastic Gravity
}

\author{
J. W. Moffat \\ Department of Physics, University of Toronto, Toronto, Ontario, Canada M5S 1A7
}

(February 7, 2008)

\begin{abstract}
Gravity is treated as a stochastic phenomenon based on fluctuations of the metric tensor of general relativity. By using a $(3+1)$ slicing of spacetime, a Langevin equation for the dynamical conjugate momentum and a FokkerPlanck equation for its probability distribution are derived. The Raychaudhuri equation for a congruence of timelike or null geodesics leads to a stochastic differential equation for the expansion parameter $\theta$ in terms of the proper time $s$. For sufficiently strong metric fluctuations, it is shown that caustic singularities in spacetime can be avoided for converging geodesics. The formalism is applied to the gravitational collapse of a star and the Friedmann-RobertsonWalker cosmological model. It is found that owing to the stochastic behavior of the geometry, the singularity in gravitational collapse and the big-bang have a zero probability of occurring. Moreover, as a star collapses the probability of a distant observer seeing an infinite red shift at the Schwarzschild radius of the star is zero. Therefore, there is a vanishing probability of a Schwarzschild black hole event horizon forming during gravitational collapse.
\end{abstract}




\section{INTRODUCTION}

Attempts to formulate a quantum theory of gravitation have not met with success to date [1]. The main attempts have been based on:

1. Perturbation theory based on expansions about Minkowski spacetime;

2. Canonical Hamiltonian formalism;

3. Path integral formalism;

4. String theory.

The first approach became untenable for two reasons: (a) The series expansions were not renormalizable for gravitational couplings to matter and beyond the first order for vacuum gravity. (b) The method chose a special frame of reference, thus failing to meet the fundamental requirements of general relativity. Approach (2) ran into problems with the Hamiltonian constraints, since one set of constraints was non-polynomial and could not be solved to yield the true gravitational degrees of freedom. Attempts to resolve this problem have met with some success, but fundamental difficulties still exist that prevent a satisfactory formulation of quantum gravity [2]. In particular, it is difficult to meet the necessary criterion that the quantum gravity theory must have a well-defined classical general relativity (GR) limit [3]. Approach (3) has met with the unresolved difficulties of defining a meaningful measure in the spacetime manifold, and the need to use an Euclidean spacetime to define the path integral. In general, it is not possible to return to curved spacetime with a local Lorentzian signature by a Wick rotation, and guarantee that the classical GR limit exists. Finally, string theory [4] has not succeeded in describing an easily interpretable solution to quantum gravity, for the theory must be formulated as a non-perturbative scheme and potential problems with causality and non-locality prevent the use of standard canonical Hamiltonian methods and other field theory techniques based on perturbation theory. 
Sakharov [5] and Jacobson [6] have formulated effective gravitational theories. Sakharov viewed spacetime as an elastic bulk system with an elasticity constant. However, this theory had difficulty yielding Newton's and Einstein's gravitational theories in a well-defined limit. Jacobson pictured Einstein's field equation as an equation of state and the gravitational variables were thermodynamic variables, so that the Einstein equation was derived from the proportionality of entropy and the black hole horizon area together with the relation $\delta Q=T d S$ connecting heat $Q$, entropy $S$, and temperature $T$. In this approach, it is not considered appropriate to quantize the classical gravitational fields as is done for the case of the electromagnetic fields.

It is well known that an analogy between classical statistical mechanics and Euclidean quantum field theory exists. This is based on the fact that the path integral measure $D \phi \exp \left(-S_{E}[\phi]\right)$ can be identified with the Boltzmann probability distribution of a statistical system, where $S_{E}[\phi]$ denotes the field theory action, which has been Wick rotated to a 4dimensional Euclidean space. The Euclidean Green functions are the correlation functions of a statistical system in thermal equilibrium. A stochastic quantization of field theory has been formulated by Parisi and $\mathrm{Wu}$ [7,8]. A new 'fictitious' time coordinate was introduced with respect to which all fields evolve, namely, the field $\phi(x)$ was supplemented with the extra coordinate $t$, such that $\phi(x) \rightarrow \phi(x, t)$, where $x^{\mu}=\left(x^{0}, x^{1}, x^{2}, x^{3}\right)$ and the fictitious time $t$ should not be confused with the Euclidean time $x^{0}$. The evolution of $\phi(x, t)$ is determined by the Langevin equation:

$$
\partial_{t} \phi(x, t)=-\left[\frac{\delta S[\phi]}{\delta \phi(x)}\right]_{\phi(x)=\phi(x, t)}+\xi_{t}(x, t),
$$

where $\xi_{t}(x, t)$ is a Markov process (Gaussian white noise). The basic assumption of stochastic quantization is that in the limit $t \rightarrow \infty$ equilibrium is reached, and that the equal time correlation functions $\phi[\eta]$ become identical to the corresponding quantum Green functions:

$$
\lim _{t \rightarrow \infty}\left\langle\phi[\eta]\left(x_{1}, t_{1}\right) \ldots \phi[\eta]\left(x_{n}, t_{n}\right)\right\rangle=\left\langle\phi\left(x_{1}\right) \ldots \phi\left(x_{n}\right)\right\rangle
$$

where the right hand-side denotes the standard vacuum expectation value in Euclidean space. 
The stochastic quantization method has been used as a way to quantize the gravitational field by Rumpf [9]. However, since to any ordinary Feynman diagram there corresponds a number of stochastic diagrams with the same topology, and it can be shown that in the equilibrium limit these stochastic diagrams exactly sum up to the corresponding Feynman diagram, then the standard problems of perturbative quantum gravity will still prevail. Moreover, there is no indication that the method alleviates any of the problems of nonperturbative quantum gravity.

Over the past few years, theoretical and experimental studies have shown that a nonlinear system in a "noisy" environment displays surprising behavior that does not conform to common intuitive experiences. Self-organization in non-equilibrium systems coupled to fluctuating environments exhibits a much richer behavior than is possible in a deterministic situation. Transition phenomena show features similar to equilibrium phase transitions and to transition phenomena encountered in non-equilibrium systems influenced by external constraints, e.g., the Bénard instability and the laser transition. Phase transition phenomena and chaotic behavior can be induced by environmental randomness 10. Statistical mechanics can display two kinds of systems: (1) a fine-tuning of parameters that leads to critical points and phase transitions and (2), self-organization which can often describe critical systems with structure spread out over every available scale [11]. These properties can be applied to an understanding of our universe at both the large and small scale [12].

In quantum gravity there is only one scale that contains Planck's constant $\hbar$, namely, the Planck length, $l_{p}=\sqrt{\hbar G}$, so that quantum gravity describes a strongly coupled phase in which there are no correlations on large scales. However, space and time can themselves be consequences of critical phenomena in which there occur fluctuations with correlations on a scale much larger than the Planck scale. Thus, there can exist a gravitational system which is weakly coupled and critical showing self-organization behavior. Such gravitational phenomena can help to remove fine-tuning behavior encountered in cosmology e.g., those associated with the initial conditions of the universe and the cosmological constant. 
It is plausible that a nonlinear gravitational system coupled to a stochastic, fluctuating spacetime geometry can lead to significant changes in the macroscopic behavior of the system, for the fluctuations could be of order $V^{0}$ and not $V^{-1}$ where $V$ denotes the characteristic volume of the system. Another way to ask this question is: do nonlinear gravitational systems, coupled to a rapidly fluctuating spacetime geometry, always adjust their macroscopic behavior to the average properties of the spacetime geometry or do there exist situations in which the system responds to the randomness of the spacetime geometry, displaying behavior forbidden under deterministic conditions?

Attempts have been made to treat the geometry of spacetime in a semi-classical way by writing Einstein's gravitational field equations in the form:

$$
G_{\mu \nu}=8 \pi G\left\langle\bar{T}_{\mu \nu}\right\rangle
$$

where $\left\langle\bar{T}_{\mu \nu}\right\rangle$ denotes the expectation value of the stress-energy tensor operator $\bar{T}_{\mu \nu}$ in a given quantum state. In order to reconcile the left hand-side of (3) with the right hand-side, it is necessary to make the gravitational field change in a discontinuous, acausal manner, a behavior unacceptable from the classical point of view.

We shall develop a probabilistic interpretation of spacetime by defining the metric tensor $g_{\mu \nu}$ as a stochastic random variable, which is defined by a set of possible values and a probability distribution $P(g)$ over this set, with $P(g) \geq 0$ and normalized to unity on its range, where $g$ denotes the metric tensor $g_{\mu \nu}$ and $P(g)$ is a scalar obtained from the contraction of a tensor quantity invariant under diffeomorphism transformations. The probability that $g$ takes a value between $g$ and $d g$ is given by $P(g) d g$. We shall use the $(3+1)$ foliation of spacetime in GR [13 [16] and the canonical formalism to derive a Langevin equation for the conjugate canonical momentum, which is treated as a random variable. A Fokker-Planck equation for the time evolution of this stochastic variable and an equation for the probability density are derived. In this formalism, we treat both the gravitational field and the matter in a probabilistic fashion, so that Eq.(3) now becomes

$$
\left\langle G_{\mu \nu}\right\rangle=8 \pi G\left\langle T_{\mu \nu}\right\rangle,
$$


and there is no logical conflict between the two sides of the equation, although the $G_{\mu \nu}$ and the $T_{\mu \nu}$ are not treated as quantum mechanical operators but instead are considered to be functions of random, stochastic variables.

In Sections II through V, we introduce basic notation for stochastic theory and probability as applied to GR, and in Section VI, we derive the Langevin equation for the conjugate momentum variable in $(3+1)$ foliated spacetime and a Fokker-Planck equation for the probability density. In Section VII, we use the Raychaudhuri equation to derive a stochastic differential equation for the expansion parameter $\theta$, and investigate the stochastic behavior of a converging congruence of geodesics. In sections VIII and IX, we apply the method to gravitational collapse and cosmology and we conclude, in Section X, with a summary of the results.

\section{METRIC FLUCTUATIONS AND STOCHASTIC PROCESSES}

Wheeler [14] suggested that quantum fluctuations in the metric tensor of spacetime, $g_{\mu \nu}$, should occur at the order of the Planck length: $l_{P}=(\hbar G)^{1 / 2}=1.6 \times 10^{-33} \mathrm{~cm}$. These fluctuations are of order:

$$
\Delta g \sim \frac{l_{P}}{L}
$$

where $L$ is the spatial dimension of the region in a local Lorentz frame of reference. Fluctuations in the curvature tensor in space are of the order:

$$
\Delta R \sim \frac{\Delta g}{L^{2}} \sim \frac{l_{P}}{L^{3}}
$$

For a domain of order $1 \mathrm{~cm}$, the curvature fluctuations are

$$
\Delta R \sim 10^{-33} \mathrm{~cm}^{-2}
$$

and for subatomic domains the metric fluctuations are $\Delta g \sim 10^{-20}$. These are so small that we can ignore them for experimentally accessible physical observations. 
Let us postulate that the spacetime geometry is a physical system. In classical GR, it is assumed that the spacetime manifold is $C^{2}$ smooth down to zero length scales. Since all known physical systems possess "noise" at some length scale, the former assumption would seem to be unrealistic. We shall also postulate that the spacetime geometry has an (unknown) underlying microscopic structure and that the subsystems of this structure undergo stochastic fluctuations at a certain length scale, which can produce macroscopic correlation lengths due to cooperative, self-organized behavior when the subsystems couple to matter.

If we treat the geometry of spacetime as a fluctuating environment, then we can consider the gravitational constant $G$ as a control parameter with the decomposition:

$$
G_{t}=G+\sigma \xi_{t}
$$

where $G$ is the Newtonian gravitational constant ( $G=6.673 \times 10^{-8} \mathrm{~g}^{-1} \mathrm{~cm}^{3} \mathrm{~s}^{-2}$ ), and $\xi_{t}$ is Gaussian white noise with $E\left\{\xi_{t}\right\}=0$ where $E$ denotes the expectation value; $\sigma$ measures the intensity of the geometrical fluctuations of the metric. Thus, $G$ will have a bell-shaped curve distribution, peaked at the average value of $G$. The central limit theorem assures that the fluctuations in $G$ are Gaussian distributed. The spacetime fluctuations of the metric are of order:

$$
\Delta g \sim \frac{\sigma \xi_{t} M}{L}
$$

Let us describe the gravitational system by a set of state variables $X$ obeying the equation $17,18,10$ :

$$
\partial_{t} X(r, t)=f_{G}(X(r, t))
$$

which describes the local evolution of $X$ in space $r$ and time $t$. The state variables $X(t)$ are constructed from the metric tensor $g$, its derivatives and possible curvature quantities, and the functionals $f_{G}(X)$ depend on the control parameter $G$. When the symmetry of the gravitating system is broken, self-organization sets in and the disorganized state becomes unstable. We assume that there exists a time independent solution $\bar{X}$ satisfying 


$$
f_{G}(\bar{X})=0,
$$

corresponding to an unorganized state. We expand $X$ about $\bar{X}$ in small perturbations:

$$
X(r, t)=\bar{X}+x(r, t),
$$

where $|x / \bar{X}| \ll 1$, and the perturbations $x(r, t)$ satisfy the equations:

$$
\partial_{t} x_{i}=\sum_{k} B_{i k} x_{k},
$$

with the solutions:

$$
x_{i}(r, t)=\sum_{k} x_{i}^{k}(r) \exp \left(\omega_{k} t\right) .
$$

The life-time of gravitational disturbances is of order

$$
\tau_{\text {macro }}=\left|\frac{1}{\operatorname{Re}\left(\omega_{\mathrm{k}}\right)}\right|,
$$

where $\tau_{\text {macro }}$ is the macroscopic time scale of evolution of the system. The $\operatorname{Re}\left(\omega_{k}\right)$ must be negative for a gravitational state $\bar{X}$ which is asymptotically stable. Gravitational transitions can occur at the point $G=G_{c}$ at which $\operatorname{Re}\left(\omega_{k}\right)$ changes sign from negative to positive values, and a new solution can occur at this point of bifurcation associated with a gravitational order parameter which vanishes at $G_{c}$.

The correlation time $\tau_{\text {cor }}$ is a measure of the rapidity of the random fluctuations of the spacetime geometry. For a stationary gravitational process it is defined by

$$
\tau_{\text {cor }}=\frac{1}{C(0)} \int_{0}^{\infty} C(\tau) d \tau,
$$

where the normalized correlation function is given by

$$
\tilde{C}(\tau)=\frac{E\left\{\delta g_{t} \delta g_{t+\tau}\right\}}{E\left\{\delta g_{t}^{2}\right\}} .
$$

For a typical exponentially decreasing correlation function,

$$
C(\tau)=C(0) \exp (-\gamma \tau),
$$


where $\gamma$ is a constant, $\tau_{\text {cor }}=1 / \gamma$. Spacetime will be characterized by rapid fluctuations with

$$
\tau_{\text {cor }} \ll \tau_{\text {macro }}
$$

For a given 3 -geometry ${ }^{(3)} \mathcal{G}$, including geometrical noise in the gravitational equations leads to the formal Langevin [19] or stochastic differential equation (SDE):

$$
\partial_{t} X\left(g_{t}\right)=f_{G_{t}}\left(g_{t}\right),
$$

where $g_{t}$ denotes the random metric tensor. Assuming for the moment that the external parameter $G_{t}$ enters in a linear way, then (20) takes the form:

$$
\partial_{t} X=f\left(g_{t}\right)+G h\left(g_{t}\right)+\sigma \xi_{t} h\left(g_{t}\right) .
$$

The extreme irregularity of Gaussian white noise means that the time derivative of the metric is not well defined. However, the standard theory of stochastic processes can handle this difficulty by defining the equivalent integral equation:

$$
X_{t}=X_{0}+\int_{0}^{t} F_{G_{t}}\left(g_{y}\right) d y+\sigma \int_{0}^{t} h\left(g_{y}\right) \xi_{y} d y,
$$

where

$$
F_{G_{t}}\left(g_{t}\right)=f\left(g_{t}\right)+G h\left(g_{t}\right) .
$$

Two definitions of the stochastic integral $\int h\left(g_{y}\right) \xi_{y} d y$ have been given by Ito [20] and Stratonovich [21]. Both of these definitions are based on the concept that integration of Gaussian white noise yields Brownian motion, which we denote by $W_{t}$, i.e., $\xi_{t}=\partial_{t} W_{t}$. Then,

$$
\int h\left(g_{y}\right) \xi_{y} d y=\int h\left(g_{y}\right) d W_{y}
$$

Both the Ito and the Stratonovich definitions of the integral lead to a consistent calculus. 


\section{ELEMENTS OF METRIC FLUCTUATION PROBABILITY THEORY}

For a manifold $\mathcal{M}$ in 4 -space, we assume the existence of a probability triple $(\Omega, \mathcal{A}, P)$ consisting of a sample space $\Omega$, a field of events $\mathcal{A}$ and a probability measure $P$ [22]. Only $\Omega$ and $\mathcal{A}$ are used to define a random variable in $\mathcal{M}$. The sample space $\Omega$ is the ensemble of elementary outcomes of spacetime measurements in $\mathcal{M}$, labeled $\omega: \omega \in \Omega$. We take it as given that the number of elementary outcomes in the manifold $\mathcal{M}$ is uncountably infinite.

The second element of the probability triple of $\mathcal{M}$ is $\mathcal{A}$ (the $\sigma$ field) of events. The set $\mathcal{A}$ satisfies the following properties:

(i) $\mathcal{A}$ contains the certain event, $\Omega \in \mathcal{A}$.

(ii) It contains the empty set $\phi: \phi \in \mathcal{A}$.

(iii) If $A$ is an event, then so also is the complement $\bar{A}=\Omega-A$.

(iv) If $A$ and $B$ are events, so is their union and intersection.

The metric tensor $g$ is defined as a random variable by the requirement that it is a function from the sample space $\Omega$ into some state space: $g: \Omega \rightarrow \mathcal{R}$. We also have

$$
A=X^{-1}((-\infty, g]) \in \mathcal{A}, \quad \forall g \in \mathcal{R}
$$

where

$$
X^{-1}(B)=\{\omega \mid g(\omega) \in B\}, \quad B \subset \mathcal{R}
$$

We define the third element $P$ as the frequency of occurrence of event $A$. Thus, on the interval $[0,1] P: \mathcal{A} \rightarrow[0,1]$. We have by definition:

$$
\begin{gathered}
P(\phi)=0, \\
P(\Omega)=1, \\
A_{n} \in \mathcal{A}, \quad A_{n} \cap_{n \neq m} A_{m}=\phi \Rightarrow P\left(\cup_{n=1}^{\infty} A_{n}\right)=\sum_{n=1}^{\infty} P\left(A_{n}\right) .
\end{gathered}
$$

The distribution function induced by the random metric variable $g$ is defined by

$$
F_{g}=P(\{\omega \mid g(\omega)\})=P(g), \quad g \in \mathcal{R}
$$


The probability density $p(g)$ is defined by

$$
F(g)=\int^{g} p(y) d y
$$

where the integral is defined as a functional integral over the metric $g$ with a given measure. In the following, it is understood that the distribution function $F_{g}$ and the probability density $p(g)$ transform as tensor quantities under diffeomorphism transformations of the coordinates.

In the standard way, we define the expectation $E\{g\}$ of the metric tensor by

$$
E\{g\}=\int_{\Omega} g d P=\int_{\Omega} g(\omega) d P(\omega)=\int_{\Omega} g(\omega) P(d \omega),
$$

and we have

$$
E\{g\}=\int_{\mathcal{R}} g p(g) d g
$$

where $E\{g\}$ is a tensor quantity. The rth moment of $g$ is

$$
E\left\{g^{r}\right\}=\int_{\Omega} g^{r}(\omega) d P(\omega)=\int_{\mathcal{R}} g^{r} d F(g)=\int_{\mathcal{R}} g^{r} p(g) d g
$$

The mean deviation of the random metric function is

$$
\sigma^{2}=E\left\{(\delta g)^{2}\right\}=E\left\{(g-m)^{2}\right\}=\int_{\mathcal{R}}(g-m)^{2} d F(g),
$$

where $m$ denotes the mean value of $g$. The number and location of the extrema of the probability density are

$$
\left.\partial_{g} p(g)\right|_{g=g_{m}}=0
$$

where $\partial_{g} p(g)$ denotes a functional derivative with respect to the metric $g$. The most probable states are given by the maxima:

$$
\left.\partial_{g g} p(g)\right|_{g=g_{m}}<0 \text {. }
$$

We shall denote stochastic processes for the metric tensor in a 3 -geometry ${ }^{(3)} \mathcal{G}$ by $g_{t}$ and deterministic metric processes by $g$. The set $\left\{g_{t} ; t \in \theta\right\}$ of real-valued random metric 
variables, $g_{t}:(\Omega, \mathcal{A}, P) \rightarrow(\mathcal{R}, \mathcal{B})$, describes a random function with the index $\theta$ and a state space $\mathcal{R}$. The index parameter will be the time and the index set $\theta$ is either the real line $\mathcal{R}$ or the non-negative half-line, if the process started at $t=0$.

The standard Wiener process $W_{t}$ has the intial condition: $W_{0}=0$. The hierarchy of probability densities for a one-dimensional fluctuating metric is

$$
\begin{gathered}
p(g, t)=(2 \pi t)^{-1} \exp \left(-g^{2} / 2 t\right) \equiv n(g, t) \\
\vdots \\
p\left(g_{1}, t_{1} ; \ldots ; g_{m}, t_{m}\right)=n\left(g_{1}, t_{1}\right) n\left(g_{2}-g_{1}, t_{2}-t_{1}\right) \ldots n\left(g_{m}-g_{m-1}, t_{m}-t_{m-1}\right) .
\end{gathered}
$$

Since the Wiener process is a Gaussian process, we define the finite dimensional probability density as Gaussian:

$$
p_{G}(g)=\left[(2 \pi)^{n} \operatorname{det} M\right]^{-1 / 2} \exp \left[-1 / 2(g-m)^{t r} M^{-1}(g-m)\right]
$$

where

$$
g^{t r}=\left(g_{1}, \ldots, g_{n}\right), \quad m^{t r}=\left(m_{1}, \ldots, m_{n}\right)
$$

and $M$ is a positive definite $n \times n$ matrix.

A stationary stochastic process in a 3 -geometry ${ }^{(3)} \mathcal{G}$ has a probability density that is invariant against shifts of time:

$$
p\left(g_{1}, t_{1} ; \ldots ; g_{n}, t_{n}\right)=p\left(g_{1}, t_{1}+t ; \ldots ; g_{n}, t_{n}+t\right)
$$

Then, we have

$$
p(g, t)=p_{S}(g),
$$

and

$$
E\left\{g_{t}\right\}=\int_{\mathcal{R}} g p(g, t) d g=\int_{\mathcal{R}} g p_{S}(g) d g=m
$$


Moreover, the two-dimensional probability density satisfies

$$
p\left(g_{1}, t_{1} ; g_{2}, t_{2}\right)=p\left(g_{1}, g_{2} ; t_{2}-t_{1}\right),
$$

so it only depends on the time difference $t_{2}-t_{1}$. The correlation function, defined by

$$
E\left\{\delta g_{t_{1}} \delta g_{t_{2}}\right\}=\int_{\mathcal{R}} \int_{\mathcal{R}}\left(g_{1}-m\right)\left(g_{2}-m\right) p\left(g_{1}, t_{1} ; g_{2}, t_{2}\right) d g_{1} d g_{2},
$$

also only depends on the time difference $t_{2}-t_{1}$.

The Gaussian white noise is very irregular but is a useful model for rapidly fluctuating phenomena. Since it has no continuous sample paths and it has infinite total power:

$$
S=2 \int_{0}^{\infty}\left(\bar{\sigma}^{2} / 2 \pi\right) d \nu=\infty,
$$

it does not occur in nature. In the idealization of $\delta$-function correlated metric fluctuations, the gravitational system will be described by an SDE or Langevin equation of the form (21), where $\sigma$ denotes the intensity of the Gaussian white noise.

A random process for the metric fluctuations is a Markov process if when the present state of the process $g_{t}$ is known, then any additional information on its past history is irrelevant for the prediction of its future evolution. The only Markov processes which have continuous and differentiable sample paths are the deterministic ones associated with classical gravity theory, given in our ${ }^{(3)} \mathcal{G}$ by

$$
\partial_{t} X(g(t))=f(g(t))
$$

with $X_{t_{0}}=I$, where the only random element is the initial value $I$. For every positive $\epsilon$ and every function of the metric, $f(g, \tau)$, there is a drift,

$$
\lim _{t \rightarrow \tau} \int_{|y-g| \leq \epsilon} p(y, t \mid g, \tau) d y=f(g, \tau) .
$$

For every positive $\epsilon$, there is a function $h(g, \tau)$ called diffusion, which satisfies

$$
\lim _{t \rightarrow \tau} \int_{|y-g| \leq \epsilon}(y-g)^{2} p(y, t \mid g, \tau) d y=h^{2}(g, \tau) .
$$

We shall also assume that there exists a positive $\delta$ such that the condition:

$$
\lim _{t \rightarrow \tau} \int_{\mathcal{R}}|y-g|^{2+\delta} p(y, t \mid g, \tau)=0,
$$

is satisfied. 


\section{THE FOKKER-PLANCK EQUATION AND STATIONARY PROBABILITIES}

The transition probability density $p(y, t \mid g, \tau)$ of the metric diffusion process $g_{t}$ satisfies the Fokker-Planck equation [23,24](FPE) or Kolmogorov forward equation [17]:

$$
\partial_{t} p(y, t \mid g, \tau)=-\partial_{y}[f(y, t) p(y, t \mid g, \tau)]+\frac{1}{2} \partial_{y y}\left[h^{2}(y, t) p(y, t \mid g, \tau)\right]
$$

Here, it is assumed that the functional derivatives occurring in (49) exist and are continuous. As in the previous sections, we have suppressed tensor indices. For time homogeneous Markov metric processes, the Fokker-Planck equation is given by

$$
\partial_{t} p(y, t \mid g)=-\partial_{y}[f(y) p(y, t \mid g)]+\frac{1}{2} \partial_{y y}\left[h^{2}(y) p(y, t \mid g)\right]
$$

Here, the drift and the diffusion are time independent. For a one-dimensional system, the FPE for the probability density becomes

$$
\partial_{t} p(g, t)=-\left[\partial_{g} f(g, t) p(g, t)\right]+\frac{1}{2} \partial_{g g}\left[h^{2}(g, t) p(g, t)\right]
$$

An important and useful solution of the FPEs can be obtained for stationary random metric processes. We expect that a gravitational system subjected to metric fluctuations for a sufficiently long time will settle down to a stationary behavior. This means that as time approaches infinity the system will reach a state for which the probability density $p_{S}(g)$ has a shape that does not change with time, i.e., the probability to find the system in the neighborhood of a particular state becomes time independent. However, the sample paths $g_{t}(\omega)$ will in general not approach a steady-state value $g_{S}(\omega)$, so that the state of the system still fluctuates. However, these fluctuations are such that $g_{t}$ and $g_{t+\tau}$ have the same probability density, $p_{S}(g)$.

The stationary solution $p_{S}(g)$ of the FPE satisfies:

$$
\partial_{t} p\left(g, t \mid g_{0}, 0\right)+\partial_{g} I\left(g, t \mid g_{0}, 0\right)=0
$$

where 


$$
I\left(g, t \mid g_{0}, 0\right)=f(g) p\left(g, t \mid g_{0}, 0\right)-\frac{\sigma^{2}}{2} \partial_{g}\left[h^{2}(g) p\left(g, t \mid g_{0}, 0\right)\right],
$$

and we have for simplicity considered the time homogeneous case. The stationary FPE is then given by

$$
\partial_{g} I_{S}(g)=0
$$

which implies that $I_{S}(g)=$ constant for $g \in\left[g_{1}, g_{2}\right]$.

The solution of the stationary FPE equation reads 10

$$
p_{S}(g)=\frac{C}{h^{2}(g)} \exp \left(\frac{2}{\sigma^{2}} \int^{g} \frac{f(x)}{h^{2}(x)} d x\right)-\frac{2}{\sigma^{2} h^{2}(g)} I \int^{g} \exp \left(\frac{2}{\sigma^{2}} \int_{y}^{g} \frac{f(x)}{h^{2}(x)} d x\right) d y,
$$

where $C$ denotes a normalization constant. When the boundaries of the gravitational system are natural, i.e., regular with instantaneous reflection imposed as a boundary condition, then there is no flow of probability out of the state space and $I=0$. By using the Ito prescription, we obtain

$$
p_{S}(g)=\frac{C}{h^{2}(g)} \exp \left(\frac{2}{\sigma^{2}} \int^{g} \frac{f(x)}{h^{2}(x)} d x\right) .
$$

In this case, the normalization constant $C$ is given by

$$
C^{-1}=\int_{g_{1}}^{g_{2}} \frac{1}{h^{2}(g)} \exp \left(\frac{2}{\sigma^{2}} \int^{g} \frac{f(x)}{h^{2}(x)} d x\right) d g<\infty .
$$

\section{NONLINEAR GRAVITATIONAL STOCHASTIC SYSTEMS}

If the gravitational equations are linear in the control parameter $G$, then we can apply directly the stochastic methods to solve for the probability density functions. However, we shall find that in certain applications the gravitational constant will enter the phenomenological equations in a nonlinear way. Consider a phenomenological equation of the form:

$$
\partial_{t} X(t)=f(X(t))+\beta(G) h(X(t))
$$


where $\beta$ is a nonlinear function of the gravitational constant $G$. For the geometrical fluctuations, we replace the constant control parameter $G$ by a stationary stochastic process $G_{t}=G+\zeta_{t}$ and form the SDE:

$$
d X_{t}=\left[f\left(X_{t}\right)+\beta\left(G+\zeta_{t}\right) h\left(X_{t}\right)\right] d t .
$$

Since nonlinear operations on generalized functions, such as the Dirac $\delta$-function, cannot be given a well-defined mathematical meaning, we cannot use a white-noise Gaussian approximation by setting $G_{t}=G+\sigma \xi_{t}$. However, methods exist which lead to satisfactory approximations to the white-noise idealization [25, 10].

We shall assume that $\zeta_{t}$ is "colored" noise and that in applications the geometry of spacetime varies on a much faster time scale than the gravitating matter system coupled to it, so that $\zeta_{t}$ is a process with a short correlation time. Let us define the geometrical fluctuations by the process $\eta_{t}$ :

$$
\eta_{t}=\beta\left(G+\zeta_{t}\right)-E\left\{\beta\left(G+\zeta_{t}\right)\right\}=\beta\left(G+\zeta_{t}\right)-m\left(G, \sigma^{2}\right) .
$$

Then we can write Eq.(59) as

$$
d X_{t}=\left[f\left(X_{t}\right)+m\left(G, \sigma^{2}\right) h\left(X_{t}\right)\right] d t+h\left(X_{t}\right) \eta_{t} d t
$$

We can "speed up" the stochastic process by writing

$$
\eta_{t}^{\epsilon}=\eta_{t / \epsilon^{2}}=\beta\left(G+\zeta_{t / \epsilon^{2}}\right)-m\left(G, \sigma^{2}\right),
$$

where $\epsilon=\tau_{\text {cor }}$ is a small parameter. Then we have

$$
d X_{t}^{\epsilon}=\left[f\left(X_{t}^{\epsilon}\right)+m\left(G, \sigma^{2}\right) h\left(X_{t}^{\epsilon}\right)\right] d t+\frac{1}{\epsilon} h\left(X_{t}\right) \eta_{t}^{\epsilon} d t
$$

and

$$
d \zeta_{t}=-\frac{1}{\epsilon^{2}} \zeta_{t} d t+\frac{\sigma}{\epsilon} d W_{t}
$$

Rescaling time, $t \rightarrow t / \tau_{\text {cor }}$, the FPE has the form: 


$$
\partial_{t} p(x, y, t)=\left(\frac{D_{1}}{\epsilon}+\frac{D_{2}}{\epsilon}+D_{3}\right) p^{\epsilon}(x, y, t)
$$

where

$$
\begin{aligned}
& D_{1}=\partial_{y} y+\frac{\sigma^{2}}{2} \partial_{y} y \\
& D_{2}=-y \partial_{x} h(x) \\
& D_{3}=-\partial_{x}\left[f(x)+m\left(G, \sigma^{2}\right) h(x)\right] .
\end{aligned}
$$

The correct SDE in the white-noise Gaussian limit is given by

$$
d X_{t}=\left[f\left(X_{t}\right)+m\left(G, \sigma^{2}\right) h\left(X_{t}\right)\right] d t+\tilde{\sigma} h\left(X_{t}\right) d W_{t}
$$

where

$$
\tilde{\sigma}^{2}=2 \int_{\mathcal{R}} p_{S}(y)\left[\beta(G+y)-m\left(G, \sigma^{2}\right)\right] \tilde{\beta}(G+y) d y
$$

and $p_{S}(x)$ is the stationary probability density, which has the form:

$$
p_{S}^{\epsilon}(x)=\frac{C}{v(x)^{2}} \exp \left(\frac{2}{\sigma^{2}} \int \frac{F(x)}{v(x) h(x)} d x\right)
$$

where

$$
F(x)=f(x)+m\left(G, \sigma^{2}\right) h(x)
$$

and

$$
v(x)=h(x)\left[1+\epsilon Q_{1}(x)+\epsilon^{2} Q_{2}(x)+\ldots\right]
$$

\section{HAMILTONIAN FORMULATION OF GRAVITY AND STOCHASTIC DIFFERENTIAL EQUATIONS}

We shall adopt the standard Lagrangian density in Einstein's gravitational theory:

$$
\mathcal{L}=\mathcal{L}_{G}+\mathcal{L}_{M}
$$


where

$$
\mathcal{L}_{G}=\sqrt{-g} g^{\mu \nu} R_{\mu \nu}
$$

$\mathcal{L}_{M}$ is the Lagrangian density for the matter field, $g=\operatorname{det} g_{\mu \nu}$ and $R_{\mu \nu}$ is the Ricci curvature tensor.

The control parameter is defined in terms of spacetime fluctuations of the gravitational constant:

$$
G_{x}=G+\sigma \xi_{x}
$$

where, as in Eq. (8), $G$ is the average value of the gravitational constant, and $\xi_{x}$ denotes Gaussian spacetime noise. The diffeomorphism invariant Einstein field equations including stochastic spacetime fluctuations take the form:

$$
G_{\mu \nu}=8 \pi\left(G T_{\mu \nu}+\sigma \xi_{x} T_{\mu \nu}\right)
$$

where $G_{\mu \nu}$ is the Einstein tensor: $G_{\mu \nu}=R_{\mu \nu}-\frac{1}{2} g_{\mu \nu} R$ and $R$ is the scalar curvature.

The first step in formulating the Hamiltonian approach to gravity is to introduce a foliation of spacetime which defines a 3 -geometry ${ }^{(3)} \mathcal{G}$. We choose a time function $t$ and a vector field $t^{\mu}$ such that the surfaces, $\Sigma$, of constant $t$ are spacelike Cauchy surfaces with $t^{\mu} \nabla_{\mu} t=1$, where $\nabla_{\mu}$ denotes the covariant differentiation with respect to the metric tensor $g_{\mu \nu}$. In contrast to Minkowski spacetime, there is no preferred coordinate system in curved spacetime. In the standard $(3+1)$ treatment of spacetime 13 16, we introduce the lapse function, $N$, by

$$
N=-g_{\mu \nu} t^{\mu} n^{\nu}=\left(n^{\mu} \nabla_{\mu} t\right)^{-1}
$$

and the shift vector $N^{\mu}$ by

$$
N^{\mu}=h^{\mu}{ }_{\nu} t^{\nu}
$$

where $n^{\mu}$ is the unit normal vector to $\Sigma$, 


$$
h_{\mu \nu}=g_{\mu \nu}+n_{\mu} n_{\nu}
$$

is the induced spatial metric on $\Sigma$, and we use the metric signature: $(-1,+1,+1,+1)$. We have

$$
n^{\mu}=\frac{1}{N}\left(t^{\mu}-N^{\mu}\right)
$$

In the following, the volume element is

$$
\sqrt{-g}=N \sqrt{h}
$$

The scalar curvature, $R$, is given by

$$
R=2\left(G_{\mu \nu} n^{\mu} n^{\nu}-R_{\mu \nu} n^{\mu} n^{\nu}\right)
$$

We also have

$$
R_{\mu \nu} n^{\mu} n^{\nu}=R_{\mu \sigma \nu}{ }^{\sigma} n^{\mu} n^{\nu}=K^{2}-K_{\mu \nu} K^{\mu \nu}-\nabla_{\mu}\left(n^{\mu} \nabla_{\sigma} n^{\sigma}\right)+\nabla_{\sigma}\left(n^{\mu} \nabla_{\mu} n^{\sigma}\right),
$$

where $K_{\mu \nu}$ is the extrinsic curvature of $\Sigma$ defined by

$$
K_{\mu \nu}=\frac{1}{2} N^{-1}\left[\partial_{t} h_{\mu \nu}-D_{\mu} N_{\nu}-D_{\nu} N_{\mu}\right]
$$

and $D_{\mu}$ is the derivative operator on the surface $\Sigma$ connected with $h_{\mu \nu}$. This leads to the expression for $\mathcal{L}_{G}$ obtained by Arnowitt, Deser, and Misner [13]:

$$
\mathcal{L}_{G}=\sqrt{h} N\left[{ }^{(3)} R+K_{\mu \nu} K^{\mu \nu}-K^{2}\right] .
$$

The Hamiltonian density associated with $\mathcal{L}_{G}$ is given by

$$
\begin{aligned}
\mathcal{H}_{G}= & \pi^{\mu \nu} \partial_{t} h_{\mu \nu}-\mathcal{L}_{G}=\sqrt{h}\left\{N\left[-{ }^{(3)} R+h^{-1} \pi^{\mu \nu} \pi_{\mu \nu}-\frac{1}{2} h^{-1} \pi_{\lambda}^{\lambda} \pi_{\sigma}^{\sigma}\right]\right. \\
& \left.-2 N_{\nu}\left[D_{\mu}\left(h^{-1 / 2} \pi^{\mu \nu}\right)\right]+2 D_{\mu}\left(h^{-1 / 2} N_{\nu} \pi^{\mu \nu}\right)\right\}
\end{aligned}
$$

where $\pi^{\mu \nu}$ is the canonically conjugate momenta to $h_{\mu \nu}$ defined by

$$
\pi^{\mu \nu}=\frac{\partial \mathcal{L}_{G}}{\partial\left(\partial_{t} h_{\mu \nu}\right)}=\sqrt{h}\left(K^{\mu \nu}-h^{\mu \nu} K\right) .
$$


In (85), the $N$ and $N_{\mu}$ are not treated as dynamical variables but, instead, define the configuration space in terms of the metric, $h_{\mu \nu}$.

Variation of

$$
H_{G}=\int_{\Sigma} \mathcal{H}_{G}
$$

leads to the constraint equations:

$$
\begin{aligned}
{ }^{(3)} R-h^{-1} \pi^{\mu \nu} \pi_{\mu \nu}+\frac{1}{2} h^{-1} \pi_{\mu}^{\mu} \pi_{\sigma}^{\sigma} & =16 \pi G T_{\perp \perp}, \\
D_{\mu}\left(h^{-1 / 2} \pi^{\mu \nu}\right) & =-8 \pi G T_{\perp}^{\nu},
\end{aligned}
$$

where $T_{\perp \perp}=T_{\mu \nu} n^{\mu} n^{\nu}$ and $T_{\perp}^{\nu}=h_{\alpha}^{\nu} T^{\alpha \beta} n_{\beta}$ are components of the stress energy-momentum tensor $T_{\mu \nu}$ for matter.

Hamilton's first order equations in time are now obtained from $H_{G}$ [13]:

$$
\begin{aligned}
\partial_{t} h_{\mu \nu}= & \frac{\delta H_{G}}{\delta \pi^{\mu \nu}}=2 h^{-1 / 2} N\left(\pi_{\mu \nu}-\frac{1}{2} h_{\mu \nu} \pi_{\sigma}^{\sigma}\right)+2 D_{(\mu} N_{\nu)} \\
\partial_{t} \pi^{\mu \nu}= & -\frac{\delta H_{G}}{\delta h_{\mu \nu}}=-N h^{1 / 2}\left({ }^{(3)} R^{\mu \nu}-\frac{1}{2}{ }^{(3)} R h^{\mu \nu}\right)+\frac{1}{2} N h^{-1 / 2} h^{\mu \nu}\left(\pi^{\sigma \tau} \pi_{\sigma \tau}-\frac{1}{2} \pi_{\lambda}^{\lambda} \pi_{\sigma}^{\sigma}\right) \\
& -2 N h^{-1 / 2}\left(\pi^{\mu \sigma} \pi_{\sigma}^{\nu}-\frac{1}{2} \pi_{\sigma}^{\sigma} \pi^{\mu \nu}\right)+h^{1 / 2}\left(D^{\mu} D^{\nu} N-h^{\mu \nu} D^{\sigma} D_{\sigma} N\right) \\
& +h^{1 / 2} D_{\sigma}\left(h^{-1 / 2} N^{\sigma} \pi^{\mu \nu}-2 \pi^{\sigma(\mu} D_{\sigma} N^{\nu)}\right)+8 \pi G T^{\mu \nu}
\end{aligned}
$$

We shall now write the stochastic differential equation for the dynamical random variable $\pi_{t}^{\mu \nu}$ as

$$
\partial_{t} \pi_{t}^{\mu \nu}=f_{t}^{\mu \nu}\left(\pi_{t}\right)+8 \pi G T^{\mu \nu}+8 \pi \sigma \xi_{t} T^{\mu \nu}
$$

where

$$
\begin{aligned}
f_{t}^{\mu \nu}\left(\pi_{t}\right)= & -N h^{1 / 2}\left({ }^{(3)} R^{\mu \nu}-\frac{1}{2}{ }^{(3)} R h^{\mu \nu}\right)+\frac{1}{2} N h^{-1 / 2} h^{\mu \nu}\left(\pi^{\sigma \tau} \pi_{\sigma \tau}-\frac{1}{2} \pi^{\mu}{ }_{\mu} \pi^{\sigma}{ }_{\sigma}\right) \\
& -2 N h^{-1 / 2}\left(\pi^{\mu \sigma} \pi^{\nu}{ }_{\sigma}-\frac{1}{2} \pi^{\lambda}{ }_{\lambda} \pi^{\mu \nu}\right)+h^{1 / 2}\left(D^{\mu} D^{\nu} N-h^{\mu \nu} D^{\sigma} D_{\sigma} N\right) \\
& +h^{1 / 2} D_{\sigma}\left(h^{-1 / 2} N^{\sigma} \pi^{\mu \nu}-2 \pi^{\sigma(\mu} D_{\sigma} N^{\nu)}\right) .
\end{aligned}
$$

Exploiting the fact that the stochastic equation is the derivative of the Wiener process, and using the control parameter, $G_{t}=G+\sigma \xi_{t}$, we obtain 


$$
d \pi_{t}^{\mu \nu}=F_{t}^{\mu \nu}\left(\pi_{t}\right) d t+B_{t}^{\mu \nu} d W_{t}
$$

where

$$
F_{t}^{\mu \nu}\left(\pi_{t}\right)=f_{t}^{\mu \nu}\left(\pi_{t}\right)+8 \pi G T^{\mu \nu}
$$

and

$$
B_{t}^{\mu \nu}=8 \pi \sigma T^{\mu \nu}
$$

The constraint equations (88a) and (88b) take the stochastic form:

$$
\begin{aligned}
{ }^{(3)} R_{t}-h_{t}^{-1} \pi_{t}^{\mu \nu} \pi_{t \mu \nu}+\frac{1}{2} h_{t}^{-1} \pi_{t \mu}^{\mu} \pi_{t \sigma}^{\sigma} & =16 \pi\left(G T_{\perp \perp}+\sigma \xi_{t} T_{\perp \perp}\right), \\
D_{\mu}\left(h_{t}^{-1 / 2} \pi_{t}^{\mu \nu}\right) & =-8 \pi\left(G T_{\perp}^{\nu}+\sigma \xi_{t} T_{\perp}^{\nu}\right) .
\end{aligned}
$$

Consider the random process

$$
Z_{t}=v\left(\pi_{t}, t\right)
$$

According to the Ito rule [17], the SDE of the process $Z_{t}$ is

$$
d Z_{t}=d v\left(\pi_{t}, t\right)=\left[\partial_{t} v(\pi, t)+F_{t}\left(\pi_{t}\right) \partial_{\pi} v(\pi, t)+\frac{1}{2} B_{t}^{2} \partial_{\pi \pi} v(\pi, t)\right] d t+B_{t} \partial_{\pi} v(\pi, t) d W_{t}
$$

where for simplicity we have written $\pi^{\mu \nu}, F^{\mu \nu}, B^{\mu \nu}$ and $T^{\mu \nu}$ as $\pi, F, B$ and $T$.

The integral form of (97) is given by

$$
\begin{aligned}
v\left(\pi_{t^{\prime}}, t^{\prime}\right)-v\left(\pi_{0}, 0\right)= & \int_{0}^{t^{\prime}}\left[\partial_{t} v\left(\pi_{t}, t\right)+F_{t}\left(\pi_{t}\right) \partial_{\pi} v\left(\pi_{t}, t\right)\right. \\
& \left.+\frac{1}{2} B_{t}^{2} \partial_{\pi \pi} v\left(\pi_{t}, t\right)\right] d t+\int_{0}^{t^{\prime}} B_{t} \partial_{\pi} v\left(\pi_{t}, t\right) d W_{t}
\end{aligned}
$$

We assume that $v(\pi, t)$ has compact support, so that

1. $v(\pi, 0)=0$,

2. $v(\pi, \infty)=0$,

3. $\int_{0}^{\infty} E\left\{\left(B_{t} \partial_{\pi} v(\pi, t)\right)^{2}\right\} d t<\infty$. 
We have

$$
E\left\{\int_{0}^{\infty} B_{t} \partial_{\pi} v\left(\pi_{t}, t\right) d W_{t}\right\}=0
$$

and for $t^{\prime} \rightarrow \infty$ in $(98)$ and taking the expectation value, we get

$$
\int_{0}^{\infty} E\left\{\left[\partial_{t} v\left(\pi_{t}, t\right)+F_{t}\left(\pi_{t}\right) \partial_{\pi} v\left(\pi_{t}, t\right)+\frac{1}{2} B_{t}^{2} \partial_{\pi \pi} v\left(\pi_{t}, t\right)\right]\right\} d t=0
$$

If the transition probability of the stochastic process $\pi_{t}$ possesses a density, then we have

$$
\int_{0}^{\infty} d t \int_{\mathcal{R}} d y p\left(y, t \mid \pi_{t}, 0\right)\left[\partial_{t} v(y, t)+F(y) \partial_{y} v(y, t)+32 \pi^{2} \sigma^{2} T^{2} \partial_{y y} v(y, t)\right]=0
$$

By performing a partial integration, we obtain

$$
\int_{0}^{\infty} d t \int_{\mathcal{R}} d y v(y, t)\left[-\partial_{t} p\left(y, t \mid \pi_{t}, 0\right)-\partial_{y}\left[F_{t}(y) p\left(y, t \mid \pi_{t}, 0\right)\right]+32 \pi^{2} \sigma^{2} T^{2} \partial_{y y} p\left(y, t \mid \pi_{t}, 0\right)\right]=0 .
$$

Since $v\left(\pi_{t}, t\right)$ is an arbitrary function, it follows from (102) that

$$
\partial_{t} p\left(y, t \mid \pi_{t}, 0\right)=-\partial_{y}\left[F_{t}(y) p\left(y, t \mid \pi_{t}, 0\right)\right]+32 \pi^{2} \sigma^{2} T^{2} \partial_{y y} p\left(y, t \mid \pi_{t}, 0\right)
$$

Thus, the probability density for the random conjugate momentum variable $\pi_{t}^{\mu \nu}$ satisfies a Fokker-Planck equation.

The stationary probability behavior of a gravitational system is given by

$$
p_{S}(\pi)=\frac{C}{64 \pi^{2} T^{2}} \exp \left(\frac{1}{32 \pi^{2} \sigma^{2}} \int^{\pi} \frac{F(u)}{T^{2}} d u\right)
$$

where $C$ is a normalization constant and we have used the Ito interpretation of (90).

\section{STOCHASTIC EQUATIONS OF MOTION}

Consider the geodesic equation:

$$
\frac{d u^{\mu}}{d s}+\Gamma_{\alpha \beta}^{\mu} u^{\alpha} u^{\beta}=0
$$

where 


$$
\Gamma_{\alpha \beta}^{\mu}=\frac{1}{2} g^{\mu \sigma}\left(\partial_{\alpha} g_{\sigma \beta}+\partial_{\beta} g_{\alpha \sigma}-\partial_{\sigma} g_{\alpha \beta}\right)
$$

denotes the Christoffel symbol, $u^{\mu}=d x^{\mu} / d s$ denotes the time-like four-velocity and $d s$ is the increment of proper time along the world line, defined by

$$
d s^{2}=-g_{\mu \nu} d x^{\mu} d x^{\nu}
$$

We can then consider $u^{\mu}$ to be a random variable, $u_{s}^{\mu}$, and form the SDE:

$$
d u_{s}^{\mu}+\Gamma_{s, \alpha \beta}^{\mu} u_{s}^{\alpha} u_{s}^{\beta} d s=-\zeta_{s} F_{s}^{\mu} d s
$$

where $F_{s}^{\mu}$ is a vector quantity random variable, formed from the metric tensor and its derivatives, $\zeta_{s}$ is a Brownian motion process in terms of the proper time $s$, and the Christoffel symbol is treated as a random variable determined by the stochastic metric $g_{s, \mu \nu}$. At the length scale for which the fluctuations of spacetime are significant, we picture a test particle moving in spacetime along a Brownian motion path such that $u_{s}^{\mu}$ does not have a well-defined derivative with respect to $s$ at a point along the world line. For larger macroscopic length scales for which the spacetime fluctuations can be neglected, the motion of the test particle becomes the same as the deterministic geodesic motion in GR.

Let us define the spatial metric:

$$
h_{\mu \nu}=g_{\mu \nu}+u_{\mu} u_{\nu}
$$

Then, $h^{\mu}{ }_{\nu}=g^{\mu \sigma} h_{\sigma \nu}$ is the projection operator onto the subspace of the tangent space perpendicular to $u^{\mu}$. The expansion $\theta$, shear $\sigma_{\mu \nu}$ and twist $\omega_{\mu \nu}$ of a congruence of geodesics are defined by

$$
\begin{aligned}
\theta & =Y^{\mu \nu} h_{\mu \nu}, \\
\sigma_{\mu \nu} & =Y_{(\mu \nu)}-\frac{1}{3} \theta h_{\mu \nu}, \\
\omega_{\mu \nu} & =Y_{[\mu \nu]},
\end{aligned}
$$

where the tensor field, $Y_{\mu \nu}$, is 


$$
Y_{\mu \nu}=\nabla_{\mu} u_{\nu}
$$

and $Y_{\mu \nu}$ is purely spatial:

$$
Y_{\mu \nu} u^{\mu}=Y_{\mu \nu} u^{\nu}=0
$$

The vector field, $u^{\mu}$, of tangents is normalized to unit length, $u^{\mu} u_{\mu}=-1$, and $\nabla_{\mu}$ is defined by

$$
\nabla_{\mu} u^{\nu}=\partial_{\mu} u^{\nu}+\Gamma_{\mu \sigma}^{\nu} u^{\sigma}
$$

The Raychaudhuri equation takes the form 26, 29, 15

$$
u^{\sigma} \nabla_{\sigma} \theta=\frac{d \theta}{d s}=-\frac{1}{3} \theta^{2}-\sigma_{\mu \nu} \sigma^{\mu \nu}+\omega_{\mu \nu} \omega^{\mu \nu}-R_{\sigma \rho} u^{\sigma} u^{\rho} .
$$

Using Einstein's field equation, we have

$$
R_{\mu \nu} u^{\mu} u^{\nu}=8 \pi G\left[T_{\mu \nu}-\frac{1}{2} g_{\mu \nu} T_{\sigma}^{\sigma}\right] u^{\mu} u^{\nu}=8 \pi G\left[T_{\mu \nu} u^{\mu} u^{\nu}+\frac{1}{2} T_{\sigma}^{\sigma}\right]
$$

We assume that the weak energy condition:

$$
T_{\mu \nu} u^{\mu} u^{\nu} \geq 0
$$

holds for matter for all timelike $u^{\mu}$. Moroever, we also assume that the strong energy condition holds:

$$
T_{\mu \nu} u^{\mu} u^{\nu} \geq-\frac{1}{2} T_{\sigma}^{\sigma}
$$

We choose the geodesic congruence to be hypersurface orthogonal, so that $\omega_{\mu \nu}=0$, whereby the third term in (114) vanishes. It follows from the condition (117) that the left hand-side of (114) is negative. Then we have

$$
\frac{d \theta}{d s}+\frac{1}{3} \theta^{2} \leq 0
$$

which gives 


$$
\frac{d}{d s}\left(\theta^{-1}\right) \geq \frac{1}{3}
$$

and, therefore,

$$
\theta^{-1}(s) \geq \theta_{0}^{-1}+\frac{1}{3} s
$$

where $\theta_{0}$ is the initial value of $\theta$. If we assume that $\theta_{0}$ is negative for a converging congruence of geodesics, then $(120)$ implies that $\theta^{-1}$ must pass through zero and $\theta \rightarrow-\infty$ within a proper time $s \leq 3 /\left|\theta_{0}\right|$. Thus, it follows for a tube of matter that at a point where $\theta \rightarrow-\infty$, the matter density $\rho \rightarrow \infty$ and there is a singularity at that point on the world line.

For null geodesics, the Raychaudhuri equation takes the form:

$$
\frac{d \theta}{d \lambda}=-\frac{1}{2} \theta^{2}-\hat{\sigma}_{\mu \nu} \hat{\sigma}^{\mu \nu}+\hat{\omega}_{\mu \nu} \hat{\omega}^{\mu \nu}-R_{\mu \nu} k^{\mu} k^{\nu}
$$

where we now consider a congruence of null geodesics with the tangent field $k^{\mu}$. The energy condition takes the form:

$$
T_{\mu \nu} k^{\mu} k^{\nu} \geq 0
$$

Let us write (114) in the form:

$$
\frac{d \theta}{d s}=-\left(\frac{1}{3} \theta^{2}+\sigma_{\mu \nu} \sigma^{\mu \nu}+8 \pi G \tilde{T}\right)
$$

where we have again chosen a congruence which is hypersurface orthogonal, $\omega_{\mu \nu}=0$, and

$$
\tilde{T}=T_{\mu \nu} u^{\mu} u^{\nu}+\frac{1}{2} T_{\sigma}^{\sigma}
$$

We can now use the stochastic control parameter, $G_{s}=G+\sigma \xi_{s}$, and obtain the SDE:

$$
d \theta_{s}=-\left(\frac{1}{3} \theta_{s}^{2}+\sigma_{s, \mu \nu} \sigma_{s}^{\mu \nu}+8 \pi G \tilde{T}\right) d s-8 \pi \sigma \tilde{T} d W_{s}
$$

where $d W_{s}$ denotes the Wiener process, $d W_{s}=d \xi_{s} d s$. For null geodesics, we obtain the SDE:

$$
d \theta_{\lambda}=-\left(\frac{1}{2} \theta_{\lambda}^{2}+\hat{\sigma}_{\lambda, \mu \nu} \hat{\sigma}_{\lambda}^{\mu \nu}+8 \pi G \tilde{T}\right) d \lambda-8 \pi \sigma \tilde{T} d W_{\lambda}
$$


We see that for a given length scale if the intensity of the fluctuations is big enough, then the left hand-sides of (125) and (126) are no longer negative definite and it no longer follows that $|\theta| \rightarrow \infty$ at points along the world line. Caustic singularities can be prevented from developing in a congruence of timelike or null geodesics if convergence occurs anywhere in the manifold, provided the geometrical fluctuations are strong enough. Thus, stochastic gravity can avoid the occurrence of singularities in spacetime. In the limit of classical GR, the Hawking-Penrose theorems hold, for the Brownian motion fluctuations of spacetime are negligible and can be neglected.

The big-bang singularity in cosmology and the singularity which occurs in the gravitational collapse of a star, in GR, can both be avoided in stochastic gravity. It is to be expected that in the limit of zero spatial dimensions and zero time, the spacetime fluctuations will dominate and smear out singularities. In the next section, we shall see that it is also possible to avoid the occurrence of infinite red shift event horizons in the collapse of stars.

\section{GRAVITATIONAL COLLAPSE}

Let us apply our stochastic gravitational theory to the problem of gravitational collapse of a star. A cooling star of mass greater than the Chandrasekhar or Oppenheimer-Volkoff mass limit cannot maintain equilibrium as either a white dwarf of a neutron star, and is predicted in GR to collapse to a black hole. We shall restrict ourselves to the case of spherically symmetric collapse of "dust" with negligible pressure, In practise, we should solve Hamilton's first order equations (89a) and (89b), since (89b) is linear in the control parameter $G$ and we can solve the stochastic equation (92) directly in the Gaussian whitenoise limit. However, instead we shall solve the collapse problem by following the treatment given by Oppenheimer and Snyder and by Weinberg [27,28].

We use a comoving coordinate system to describe the freely falling dust particles. The metric is given in Gaussian normal form by

$$
d s^{2}=d t^{2}-U(r, t) d r^{2}-V(r, t)\left(d \theta^{2}+\sin ^{2} \theta d \phi^{2}\right)
$$


while the energy-momentum tensor for the fluid is

$$
T^{\mu \nu}=\rho u^{\mu} u^{\nu}
$$

where $\rho(r, t)$ is the proper energy density and $u^{\mu}$ is given in comoving coordinates by

$$
u^{r}=u^{\theta}=u^{\phi}=0, \quad u^{0}=1 .
$$

The energy conservation equation is

$$
\partial_{t}(\rho V \sqrt{U})=0
$$

The Einstein field equations are given by

$$
R_{\mu \nu}=8 \pi G N_{\mu \nu}
$$

where

$$
N_{\mu \nu}=T_{\mu \nu}-\frac{1}{2} g_{\mu \nu} T_{\sigma}^{\sigma}=\rho\left(\frac{1}{2} g_{\mu \nu}+u_{\mu} u_{\nu}\right)
$$

Assuming that the collapse is homogeneous, we can seek a separable solution:

$$
U=R^{2}(t) f(r), \quad V=S^{2}(t) g(r)
$$

Then, Einstein's field equations require that 28] $\dot{S} / S=\dot{R} / R$, where $\dot{R}=\partial_{t} R$. We can choose : $S(t)=R(t)$ and redefine the radial coordinate, so that $V=R^{2}(t) r^{2}$. Solving the resulting Einstein field equations leads to the Friedmann-Robertson-Walker (FRW) metric:

$$
d s^{2}=d t^{2}-R^{2}(t)\left[\frac{d r^{2}}{1-k r^{2}}+r^{2} d \theta^{2}+r^{2} \sin ^{2} \theta d \phi^{2}\right]
$$

We normalize $R(t)$ so that $R(0)=1$ and obtain:

$$
\rho(t)=\rho(0) R^{-3}(t)
$$

The field equations yield

$$
\dot{R}^{2}(t)=\frac{8 \pi G}{3} \frac{\rho(0)}{R(t)}-k,
$$


where $k$ is a constant given by

$$
k=\frac{8 \pi G}{3} \rho(0)
$$

Eq.(136) becomes

$$
\dot{R}^{2}=k\left[\frac{1}{R(t)}-1\right]
$$

with the parametric cycloid solution:

$$
\begin{aligned}
t & =\left(\frac{\psi+\sin \psi}{2 \sqrt{k}}\right), \\
R & =\frac{1}{2}(1+\cos \psi) .
\end{aligned}
$$

At time $t=t_{S}$, where

$$
t_{S}=\frac{\pi}{2 \sqrt{k}}=\frac{\pi}{2}\left(\frac{3}{8 \pi G \rho(0)}\right)
$$

the fluid sphere collapses to an infinite proper energy density. The resulting singularity as the end stage of collapse is inevitable in classical GR, as follows from the Hawking-Penrose theorems on gravitational collapse [29].

According to the Birkhoff theorem, the metric outside the collapsing star is static and given by the Schwarschild solution:

$$
d s^{2}=\left(1-\frac{2 G M}{r}\right) d t^{2}-\left(1-\frac{2 G M}{r}\right)^{-1} d r^{2}-r^{2}\left(d \theta^{2}+\sin ^{2} \theta d \phi^{2}\right) .
$$

By choosing an integrating factor, we can transform the metric to a standard form [28]:

$$
d s^{2}=B(r, t) d t^{2}-A(r, t) d r^{2}-r^{2}\left(d \theta^{2}+\sin ^{2} \theta d \phi^{2}\right),
$$

where

$$
\begin{aligned}
& B=\frac{R}{S}\left(\frac{1-k r^{2}}{1-k a^{2}}\right)^{1 / 2} \frac{\left(1-k a^{2} / S\right)^{2}}{\left(1-k r^{2} / R\right)}, \\
& A=\left(1-\frac{k r^{2}}{R}\right)^{-1}
\end{aligned}
$$


and the constant $a$ is equated to the radius of the sphere in comoving polar coordinates. It follows that the interior and the exterior solutions match continuously at $r=a R(t)$ when

$$
k=\frac{2 G M}{a^{3}},
$$

which gives $M=\frac{4 \pi}{3} \rho(0) a^{3}$.

Let us rewrite Eq.(138) as

$$
\dot{R}(t)=-\left(\frac{2 G M}{a^{3}}\right)^{1 / 2}\left[\frac{1}{R(t)}-1\right]^{1 / 2}
$$

where for the collapse problem we have chosen the negative square root. The nonlinear SDE has the form:

$$
d R_{t}=\left[\sqrt{G} f\left(R_{t}\right)+\beta\left(\sqrt{G}+\zeta_{t}\right) f\left(R_{t}\right)\right] d t
$$

where

$$
f\left(R_{t}\right)=-\left(\frac{2 M}{a^{3}}\right)^{1 / 2}\left(\frac{1}{R_{t}}-1\right)^{1 / 2}
$$

For rapidly varying geometrical fluctuations, corresponding to a random process with a $\zeta_{t}$ that has a short correlation time, we can obtain in the white-noise limit the approximate stationary probability density:

$$
p_{S}(R)=\frac{C}{v^{2}(R)} \exp \left[\frac{2 \sqrt{G}}{\sigma^{2}} \int^{R} \frac{d R}{v(R)}\right]
$$

where $C$ is a normalization constant and

$$
v(R)=f(R)\left[1+\tau_{\text {cor }} Q_{1}(R)+\ldots\right] .
$$

To lowest order we get

$$
p_{S}(R) \sim C\left(\frac{a^{3}}{2 M}\right)\left(\frac{R}{1-R}\right) \exp \left\{\frac{2 \sqrt{G}}{\sigma^{2}}\left(\frac{a^{3}}{2 M}\right)^{1 / 2}[\sqrt{R} \sqrt{1-R}-\arcsin \sqrt{R}]\right\} .
$$

We see that $R=1$ is a natural boundary [17]: both the drift and the diffusion coefficients vanish at $R=1$. For $\sqrt{G}<\sigma^{2} / 2\left(a^{3} / 2 M\right)^{1 / 2}$ the point $R=1$ is attracting and the 
stationary probability is mostly concentrated at $R=1$. The point $\sqrt{G}=\sigma^{2} / 2\left(a^{3} / 2 M\right)^{1 / 2}$ is a transition point, since for $\sqrt{G}>\sigma^{2} / 2\left(a^{3} / 2 M\right)^{1 / 2}$ the probability density moves away from the divergent point $R=1$ and a new stationary probability density emerges with a large finite probability for collapse towards $R=0$. However, for $R \rightarrow 0$, we have

$$
p_{S}(R) \sim 0
$$

We therefore arrive at the result that as the star collapses there is zero probability for $R(t)$ to have the value zero, and consequently there is zero probability of having a singularity as the final state of collapse.

Let us now consider the red shift emitted from the surface of the star as it collapses. The fractional change of wave length emitted at the surface is

$$
z=\frac{\lambda^{\prime}-\lambda_{0}}{\lambda_{0}}=\frac{d t^{\prime}}{d t}-a \dot{R}(t)\left(1-\frac{2 G M}{a R(t)}\right)^{-1}-1
$$

where

$$
t^{\prime}=t+\int_{a R(t)}^{t}\left(1-\frac{2 G M}{r}\right)^{-1} d r
$$

is the time it takes for a light signal emitted in a radial direction at standard time $t$ to reach a distant point $r$. In the limit, $R(t) \rightarrow 2 G M / a=k a^{2}$, we obtain by using (145):

$$
z=2\left(1-\frac{k a^{2}}{R(t)}\right)^{-1}
$$

We have

$$
d z=f(G, R) d t
$$

where

$$
\begin{aligned}
f(G, R) & =-\frac{4 G M}{a}\left(1-\frac{2 G M}{a R}\right)^{-2} \frac{\dot{R}}{R^{2}} \\
& =\left(\frac{2}{a}\right)^{5 / 2}(G M)^{3 / 2}\left(1-\frac{2 G M}{a R}\right)^{-2} \frac{(1-R)^{1 / 2}}{R^{5 / 2}}
\end{aligned}
$$

We can now define the nonlinear SDE: 


$$
d z_{t}=\mu(G) u\left(R_{t}\right) d t+\gamma\left(G+\zeta_{t}\right) u\left(R_{t}\right) d t
$$

where the function $u(R) \rightarrow \infty$ as $R \rightarrow 2 G M / a$, corresponding to the infinite red shift limit as the event horizon is approached during collapse predicted by classical GR, and $\zeta_{t}$ is a short time stochastic, colored process. Expanding around the white-noise Gaussian limit, we can derive the approximate stationary probability density for the red shift in the limit $R \rightarrow 2 G M / a:$

$$
p_{S}(z) \sim \frac{C}{u^{2}(R)} \exp \left[\frac{2 \mu(G)}{\sigma^{2}} \int \frac{d R}{u(R)}\right] .
$$

The result emerges from (158) that provided the bound

$$
\int \frac{d R}{u(R)}<\frac{\sigma^{2}}{\mu(G)} \ln (u)
$$

is satisfied, then

$$
p_{S}(z) \sim 0
$$

and the probability for an infinite red shift at $R=2 G M / a$ is zero. Thus, in our stochastic gravitational theory the probability for a black hole event horizon with an infinite red shift to form during gravitational collapse is zero when (159) is satisfied. On the other hand, once a trapped surface forms in classical GR collapse, then the red shift seen by a distant observer must inevitably become infinite at the radius $r_{G}=2 G M$. We recall that for physical collapse in nature, the unphysical regions which occur in the Penrose diagram for the extended (Kruskal) Schwarzschild solution are covered up by the collapsing matter. In GR, the metric outside the collapsing star must be the Schwarzschild static metric (Birkhoff theorem).

In stochastic gravity, the geometrical fluctuations about the deterministic event horizon cut off the high wave length at a finite value, $\lambda=\lambda_{c}$, when (159) holds, as viewed by an observer at large distances from the collapsing star. In fact, the distant observer does not see a collapsed object described by a static Schwarzschild metric with no hair. The collapsed object will fluctuate about the average Schwarzschild solution with a characteristic time 
determined by the size of the correlation function for the Schwarzschild metric. The red shift of the collapsed object can be high, so that an outside observer would believe that it is a black hole. The cooperative effects associated with the self-organising microscopic subsystems comprising the event horizon can produce a large or even infinite correlation length for the microscopic fluctuations, cutting off the infinite wavelength radiation emitted by the macroscopic surface of the collapsing star as $R \rightarrow 2 G M / a$.

Our use of the stationary probability density for the collapse problem means that we have assumed that after an infinite time has elapsed the system evolves to a stationary state. This is compatible with the fact that the spherically symmetric collapse problem has well-defined flat space asymptotic limits as $r \rightarrow \infty$. Since in stochastic gravity, it can take a long (but finite) time for a distant observer to see the formation of an apparent event horizon during collapse, this is consistent with the observer measuring the stationary probability density $p_{S}$ as the limit $p(z) \rightarrow p_{S}(z)$ as $t \rightarrow \infty$.

We have succeeded in removing both the singularity in collapse and the black hole event horizon in our stochastic probability gravitational theory (provided (159) is satisfied.) The deterministic GR predictions are clearly obtained in the limit that the intensity of fluctuations vanishes. As we have seen in the last section, the results are consistent with the Hawking-Penrose theorems for collapse, because for large enough fluctuations of the stochastic metric, $g_{s, \mu \nu}$, caustics can be prevented from occurring for converging congruences of timelike or null geodesics in the spacetime manifold.

The 'greyness' of the dense collapsed object depends on the size of the correlation time $\tau_{\text {cor }}$. The correlation length can be significantly larger than the Planck length scale, $l_{P}$, since the fluctuations are associated with a stochastic property of the gravitational field, which is not directly proportional to Planck's constant $\hbar$, and is therefore not strongly correlated to regions at the scale of $l_{P}$. If the dense collapsed object is sufficiently grey, then this could solve the information loss problem first introduced by Hawking [30]. Again, this depends on the size of the correlation length associated with the geometrical fluctuations. Since the deterministic event horizon has been removed, either there is no Hawking radiation emitted 
from the surface of the collapsed object, or the spectrum of the radiation is significantly modified from its behavor in deterministic GR. This raises the question: will black holes form in the collapse of astrophysical objects in our statistical mechanical treatment of gravity? If the answer is no, then what kind of object would describe the final state of collapse? These interesting questions require further investigation, before any conclusions can be reached about this fundamental problem.

\section{THE COSMOLOGICAL BIG-BANG SINGULARITY}

Let us now apply our stochastic formalism to cosmology. We shall consider the standard FRW cosmology. The FRW metric has the form (134) and solving Einstein's field equations including the cosmological constant term yields the Friedmann equation:

$$
\dot{R}^{2}(t)=\frac{8 \pi G}{3} R^{2}(t)\left(\frac{\rho_{0, M}}{R^{3}(t)}+\frac{\rho_{0, r}}{R^{4}(t)}\right)+\frac{\Lambda}{3} R^{2}(t)-k
$$

where $R(t)$ denotes the cosmic scale factor, $k$ now denotes the measure of the spatial curvature: $k=1,0,-1, \rho_{0, M}$ and $\rho_{0, r}$ denote the present density of matter and radiation, respectively, and $\Lambda$ denotes the cosmological constant.

Let us consider for simplicity the case of zero spatial curvature and zero cosmological constant, $\Lambda=k=0$. We shall focus on the radiation dominated universe near $t=0$. Then, Eq.(161) can be written as

$$
d R=\sqrt{G}\left(\frac{8 \pi \rho_{0, r}}{3}\right)^{1 / 2} \frac{1}{R^{2}} d t
$$

We can now form the nonlinear SDE:

$$
d R_{t}=\sqrt{G} f\left(R_{t}\right) d t+\alpha\left(\sqrt{G}+\zeta_{t}\right) f\left(R_{t}\right) d t
$$

where $\zeta_{t}$ is assumed to be a short correlation time stochastic process associated with colored fluctuations of the geometry, and

$$
f\left(R_{t}\right)=\left(\frac{8 \pi \rho_{0, r}}{3}\right)^{1 / 2} \frac{1}{R_{t}^{2}} .
$$


The approximate stationary probability density calculated in the limit of white-noise is given by

$$
p_{S}(R) \sim C\left(\frac{3 R^{4}}{8 \pi \rho_{0, r}}\right) \exp \left[\left(\frac{2 \sqrt{G}}{\sigma^{2}}\right)\left(\frac{1}{24 \pi \rho_{0, r}}\right)^{1 / 2} R^{3}\right]
$$

where $C$ is a normalization constant. We have $R \rightarrow 0$ as $t \rightarrow 0$ and we see that the probability of $R$ reaching $t=0$ is zero. Thus, in our stochastic gravity the probability of the big-bang singularity occurring is zero. As in the case of gravitational collapse, the fluctuations of the geometry near $t=0$ smear out the singularity. The result is consistent

with the Hawking-Penrose theorem [29], which states that in classical GR, the universe must collapse to a singularity at $t=0$ provided the positive energy conditions are satisfied, because for large enough fluctuations in the neighborhood of $t=0$, the converging geodesic congruences can be prevented from forming a singularity, as has been demonstrated in section VII.

\section{CONCLUSIONS}

We have formulated a self-consistent gravitational theory based on stochastic diffusion processes. The geometry of spacetime is assumed to be like a fluctuating environment with the control parameter, $G_{x}=G+\sigma \xi_{x}$. The fluctuations of the control parameter form a bell-shaped distribution about the deterministic Newtonian gravitational constant $G$. This theory represents a statistical mechanical treatment of gravity with a clearly determined classical GR limit when the intensity of the spacetime fluctuations vanishes. In this approach, Einstein's gravitational theory is a deterministic "effective" theory, which holds to a very good approximation for macroscopic gravitational systems with scales larger than the correlation lengths associated with the geometrical fluctuations. The stochastic gravity theory does not represent a complete quantum mechanical theory and cannot solve the problem of quantum gravity, but it does lead to a methodology that can account for the complexity of gravitational phenomena as are experienced in cosmology, gravitational collapse and 
astrophysics. Since it is a probabilistic theory, it reconciles a probabilistic interpretation of the energy-stress tensor of matter and the geometry of spacetime. It leads to critical phenomena and self-organization of gravitational systems, which are strongly expected to play a significant role in astrophysics and cosmology [31].

By working with a $(3+1)$ foliation of spacetime, and using the Arnowitt, Deser and Misner formalism, we were able to obtain a stochastic differential equation for the random canonically conjugate momentum $\pi_{t}^{\mu \nu}$. We also derived a Fokker-Planck equation for the probability density of $\pi_{t}^{\mu \nu}$ and showed how a stationary probability density for the dynamical variable $\pi_{t}^{\mu \nu}$ could be obtained.

Stochastic geodesic congruences were studied and from the Raychaudhuri equation, we derived a stochastic differential equation for the expansion parameter $\theta$, which led to the result that, assuming the weak and strong positive energy conditions are satisfied and for sufficiently strong spacetime fluctuations at small scales, a congruence of converging geodesics can be prevented from forming caustic singularities in the manifold, thereby avoiding the classical GR Hawking-Penrose singularity theorems.

For certain applications of the formalism, we found it necessary to consider nonlinear non-Markovian colored processes which were expanded about the white-noise Gaussian limit, a limit expected to hold for a rapidly fluctuating spacetime geometry. Stationary state probability densities were obtained in the Gaussian limit with a short correlation time for the fluctuating geometry in gravitational collapse and cosmology. It was found that the fluctuations of the geometry smeared out the singularity in gravitational collapse. Similarly, the big-bang singularity was smeared out in a probabilistic sense, leading to a singularity-free FRW universe.

A stochastic treatment of gravitational collapse resulted in the removal of black hole event horizons (given that the bound (159) is satisfied) by cutting off the high frequencies in the infinite red shift limit. This means that either there is no Hawking radiation emitted from the surfaces of the collapsed objects, or the blackbody radiation spectrum predicted by deterministic GR is significantly modified. Moreover, the lack of a rigorous event horizon 
could remove the problem of information loss [30] depending on the 'greyness' of the collapsed objects.

We can also extend the stochastic methods developed here to the nonsymmetric gravitational theory (NGT) [32], so that for small enough fluctuations of the non-Riemannian geometry we obtain the classical NGT theory.

\section{ACKNOWLEDGMENTS}

I thank M. Clayton, N. Cornish, L. Demopolous, J. Légaré, J. Preskill, and P. Savaria for helpful and stimulating discussions. This work was supported by the Natural Sciences and Engineering Research Council of Canada. 


\section{REFERENCES}

[1] For a recent review of quantum gravity, see: C. Isham, "Structural Issues in Quantum Gravity", gr-qc/9510063 1996, to be published in the proceedings of the GR14 Conference.

[2] A. A. Ashtekar, Phys. Rev. D36, 1587 (1987); Non-perturbative Canonical Gravity, World Scientific, Singapore, 1991.

[3] L. Smolin, "The Classical Limit and the Form of the Hamiltonian Constraint in Nonperturbative Quantum General Relativity", gr-qc/9609034 (1996).

[4] M. B. Green, J. H. Schwarz, and E. Witten, Superstring Theory, Vol. I, Cambridge University Press, 1987.

[5] A.D. Sakharov, Doklady Akad. Nauk S.S.S.R. 177, 70 (1967). English translation in Sov. Phys. Doklady 12, 1040 (1968).

[6] T. Jacobson, Phys. Rev. Letts. 75, 1260 (1995).

[7] G. Parisi and Wu Yongshi, Sci. Sin. 24, 483 (1981).

[8] Stochastic Quantization, editors P. H. Damgaard and H. Hüffel, World Scientific, Singapore, 1987.

[9] H. Rumpf, Phys. Rev. D33, 942 (1986).

[10] W. Horsthemke and R. Lefever, Noise-Induced Transitions, Theory and Applications in Physics, Chemistry, and Biology, Springer-Verlag, Berlin, Heidelberg, New York, 1984.

[11] P. Bak, C. Tang and K. Wiesenfeld, Phys. Rev. A38, 364 (1988); P. Bak, How Nature Works, Springer-Verlag New York, Inc. 1996.

[12] L. Smolin, "Cosmology as a Problem in Critical Phenomena", Proceedings of the Guanauato Conference on Self-organization and Binary Networks, Springer, 1995. 
[13] R. Arnowitt, S. Deser, and C. W. Misner, "The Dynamics of General Relativity", in Gravitation: an Introduction to Current Research, editor L. Witten, J. Wiley, New York, 1962.

[14] C. Misner, K. S. Thorne, and J. A. Wheeler, Gravitation, J. Freeman, San Francisco, 1973.

[15] M. Wald, General Relativity, University of Chicago Press, Chicago and London, 1984.

[16] Y. Choquet-Bruhat and J. W. York, Jr. General Relativity and Gravitation, editor A. Held, Plenum Press, 1980.

[17] I. I. Gihman and A. V. Skorohod, Stochastic Differential Equations, Springer-Verlag, Berlin, Heidelberg, New York, 1985.

[18] B. Øksendal, Stochastic Differential Equations, an Introduction with Applications, Springer, Berlin, Heidelberg, New York, 1995.

[19] P. Langevin, C. R. Acad. Sci. (Paris) 146, 530 (1908).

[20] K. Ito, Proc. Imp. Acad. 20, 519 (1944).

[21] L. Stratonovich, Conditional Markov Processes and Their Applications to the Theory of Optimal Control, Elsevier, New York, 1968.

[22] J. Pitman, Probability, Springer, Berlin, Heidelberg, New York, 1992.

[23] A. D. Fokker, Ann. Phys. 43, 810 (1914).

[24] M. Planck, Sitzber. Pr. Akad. Wiss. P. 324 (1917).

[25] M. San Miguel and J. M. Sancho, J. Stat. Phys. 22, 605 (1980); "Muiltiplicative Ornstein-Uhlenbeck Noise in Non-equilibrium Systems", in Stochastic Nonlinear Systems in Physics, Chemistry, and Biology, Springer series: Synergetics, Vol. 8, edited by L. Arnold and R. Lefever, Springer-Verlag, Berlin, Heidelberg, New York, 1981. 
[26] A. Raychaudhuri, Phys. Rev. 98, 1123 (1955).

[27] J. R. Oppenheimer and H. Snyder, Phys. Rev. 56, 455 (1939).

[28] S. Weinberg, Gravitation and Cosmology: Principles and Applications of the General Theory of Relativity, John Wiley, New York, 1972.

[29] S. W. Hawking and G. F. R. Ellis, The Large Scale Structure of Space-Time, Cambridge University Press, Cambridge, 1973.

[30] S. W. Hawking, Phys. Rev. D14, 2460 (1976); Commun. Math. Phys. 87, 395 (1982).

[31] J. W. Moffat, "A Self-Organized Critical Universe", University of Toronto preprint UTPT-97-03; gr-qc/9702014, 1997.

[32] J. W. Moffat, J. Math. Phys. 36, 5897 (1995). 